This is the author's copy of the publication as archived with the DLR's electronic library at http://elib.dlr.de. Please consult the original publication for citation, see e.g. https://ieeexplore.ieee.org/document/9438423

\title{
Locomotion Control Functions for the Active Chassis of the MMX Rover
}

\section{J. Skibbe and S. Barthelmes and F. Buse}

JAXA's Martian Moons eXploration Mission (MMX) includes the delivery of an exploration rover to the Mars moon Phobos in 2026, engineered by the Centre National d'Études Spatiales (CNES) and the German Aerospace Center (DLR). On Phobos, the gravity is about two thousand times lower than on Earth, which is why it is also called a milli-g environment. While the actual surface of Phobos is largely unknown, it is agreed within mission team that areas covered with regolith are to be expected. The design of the rover includes four actuated legs, with one rotational degree of freedom (DOF) each, and four individually driven, non-steerable wheels. The first task of the rover after landing on the surface of Phobos will be to deploy itself from its cruise configuration and to stand up on its wheels. Due to the rover dimensions, a full rotation of the legs and a wheel slip compensation are essential for this task. During operations, the locomotion system needs to provide drive and steer, as well as point turn abilities. Furthermore, the solar panel sun pointing, as well as the adjustment of scientific instruments, require that the rover aligns its body orientation and alters its body-to-ground distance. To satisfy all these requirements to the locomotion system of the MMX rover, appropriate locomotion control functions were developed. For driving curves and performing point turns, the skid steering method is applied and an "inching" locomotion mode for especially soft and steep terrain is adapted. In this inching locomotion, the front and rear wheel pair move alternately while the rover body moves up and down, which leads to enhanced traction performance compared to conventional driving in soft sand. This implies lower wheel slippage and sinkage resulting in a higher safety of the full rover system. The body orientation function, which is based on a kinematic control as well, provides a well coordinated movement and zero longitudinal slippage of the wheels during its execution. In this paper, a detailed description of the control algorithms is given and results from lab tests are presented and discussed. In a successful mission, these locomotion control functions will be the first ones actuating a wheeled mobile robot in milli-g environment.

\section{Copyright Notice}

C2020 IEEE. Personal use of this material is permitted. Permission from IEEE must be obtained for all other uses, in any current or future media, including reprinting/republishing this material for advertising or promotional purposes,creating new collective works, for resale or redistribution to servers or lists, or reuse of any copyrighted component of this work in other works.

J. Skibbe, S. Barthelmes and F. Buse, "Locomotion Control Functions for the Active Chassis of the MMX Rover," 2021 IEEE Aerospace Conference (50100), 2021, pp. 1-9, doi: 10.1109/AERO50100.2021.9438423. 


\section{Locomotion Control Functions for the Active Chassis of the MMX Rover}

\author{
Juliane Skibbe \\ German Aerospace Center (DLR) \\ Münchener Str. 20 \\ 82234 Weßling, Germany \\ Juliane.Skibbe@dlr.de
}

\author{
Stefan Barthelmes \\ German Aerospace Center (DLR) \\ Münchener Str. 20 \\ 82234 Weßling, Germany \\ Stefan.Barthelmes@dlr.de
}

\author{
Fabian Buse \\ German Aerospace Center (DLR) \\ Münchener Str. 20 \\ 82234 Weßling, Germany \\ Fabian.Buse@dlr.de
}

\begin{abstract}
JAXA's Martian Moons eXploration Mission (MMX) includes the delivery of an exploration rover to the Mars moon Phobos in 2026, engineered by the Centre National d'Études Spatiales (CNES) and the German Aerospace Center (DLR). On Phobos, the gravity is about two thousand times lower than on Earth, which is why it is also called a milli-g environment. While the actual surface of Phobos is largely unknown, it is agreed within mission team that areas covered with regolith are to be expected. The design of the rover includes four actuated legs, with one rotational degree of freedom (DOF) each, and four individually driven, non-steerable wheels. The first task of the rover after landing on the surface of Phobos will be to deploy itself from its cruise configuration and to stand up on its wheels. Due to the rover dimensions, a full rotation of the legs and a wheel slip compensation are essential for this task. During operations, the locomotion system needs to provide drive and steer, as well as point turn abilities. Furthermore, the solar panel sun pointing, as well as the adjustment of scientific instruments, require that the rover aligns its body orientation and alters its body-to-ground distance. To satisfy all these requirements to the locomotion system of the MMX rover, appropriate locomotion control functions were developed. For driving curves and performing point turns, the skid steering method is applied and an "inching" locomotion mode for especially soft and steep terrain is adapted. In this inching locomotion, the front and rear wheel pair move alternately while the rover body moves up and down, which leads to enhanced traction performance compared to conventional driving in soft sand. This implies lower wheel slippage and sinkage resulting in a higher safety of the full rover system. The body orientation function, which is based on a kinematic control as well, provides a well coordinated movement and zero longitudinal slippage of the wheels during its execution. In this paper, a detailed description of the control algorithms is given and results from lab tests are presented and discussed. In a successful mission, these locomotion control functions will be the first ones actuating a wheeled mobile robot in milli-g environment.
\end{abstract}

\section{TABle of Contents}

1. INTRODUCTION............................

2. StATE-of-the-ART $\ldots \ldots \ldots \ldots \ldots \ldots \ldots \ldots \ldots \ldots . \ldots \ldots$

3. Locomotion Control Algorithm ........... 2

4. Testing, Results And Discussion ..........6

5. Conclusion $\ldots \ldots \ldots \ldots \ldots \ldots \ldots \ldots \ldots \ldots \ldots . \ldots \ldots$

REFERENCES ...............................9

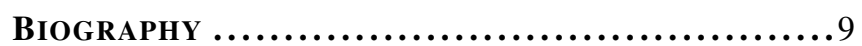

\section{INTRODUCTION}

The MMX rover is a contribution to JAXA's Martian Moons eXploration mission and is jointly developed by DLR and CNES. The rover is deployed as a surface scout, before the

978-1-7281-7436-5/21/\$31.00 (c)2021 IEEE

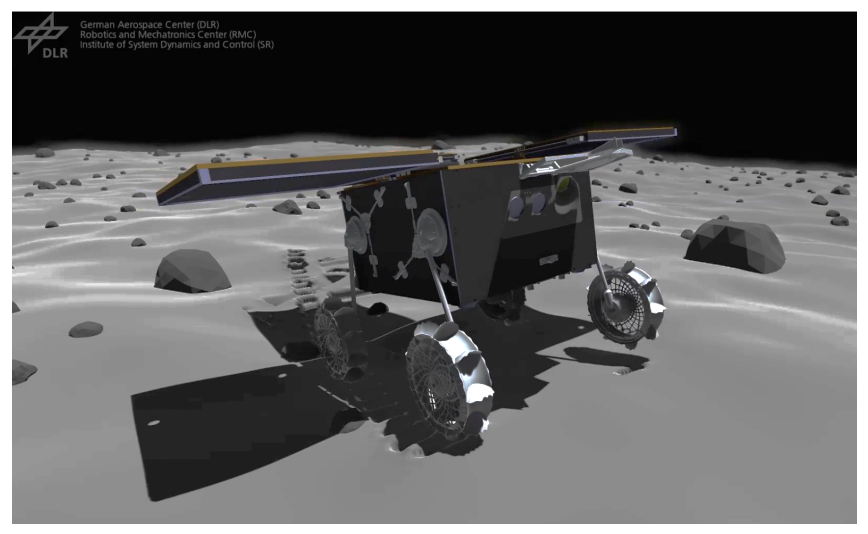

Figure 1: The MMX rover in simulation.

landing of the main spacecraft, to assess properties of the ground by means of several cameras and accelerometers. Besides that main mission goal, DLR and CNES set themselves further scientific and technological objectives that are only reachable by in-situ science, see [1] for further details. Since one of these goals is the characterization of driving abilities on a milli-g body, the MMX rover is designed as a wheeled rover, see figure 1. More specifically, it has four wheels, each of them mounted on a rotatable leg to allow for a folded position in the spacecraft and a deployment, also called uprighting, once the rover has landed on Phobos. These eight rotational degrees of freedom are driven individually by a custom-made and highly integrated drive train, see [2] for details on the mechatronic design of this locomotion subsystem.

Driven by different stakeholder expectations and science instrument requirements, high-level requirements can be derived for the design of the locomotion modes:

- The rover shall upright itself to stand on its wheels and be able to deploy its solar generators once it has landed on Phobos.

- The rover shall be able to drive straight, in curves and turn on the spot to be able to maneuver in the unknown environment that it will land in.

- The rover shall be able to increase and decrease its bodyto-ground clearance, e.g. for enabling optical focusing of the on-board Raman spectrometer, see [1].

- The rover shall be able to adjust its orientation with respect to the ground, e.g. to point to the sun for an increased power generation of the solar arrays.

These requirements can be met by means of four different locomotion modes. Firstly, an uprighting mode uses precomputed leg angles from another software module, which is dedicated to performing the uprighting autonomously. The 
chassis controller deduces the correct wheel movement from the leg motion and commands both to the wheel and shoulder motors. Secondly, a drive mode realizes straight driving, as well as curve driving and point turning, through a geometric skid steering approach. Thirdly, an alignment mode computes the coordinated leg angles for achieving an altered body height and orientation through the rover kinematics. Ultimately, a fourth mode, the so-called inching locomotion, is implemented to drive on particularly soft sand as well as steep slopes with an improved performance.

These four locomotion modes are developed and will be implemented on the on-board computer (OBC). More specifically, this chassis controller is part of the locomotion software partition that takes care of - among others - communication drivers, housekeeping, locomotion subsystem startup and shutdown as well as error handling.

In section 2, the state of the art for planetary rovers with similar rover architecture, their capabilities and control strategies are briefly reviewed. The main scope of this paper is the detailed explanation of the core algorithms of the four locomotion modes. Therefore, in section 3, the underlying kinematic algorithms to meet the locomotion requirements are explained in some detail. Finally, in section 4, the algorithms are verified by means of lab tests with a first prototype of the locomotion subsystem including a preliminary performance assessment of the system.

\section{STATE-OF-THE-ART}

Many different chassis designs for rovers for in-situ exploration exist. SEENI et al. [3] reviewed robotic mobility systems for planetary surface exploration until 2010. Due to their simplicity, the most common kind of locomotion is the one with wheels. Many wheeled planetary rovers landed on Mars and Moon to date [4], but the MMX rover will be the first wheeled rover driving under milli-g conditions and also the first wheeled one with an active chassis participating in a space mission. Most of the Mars and Moon rovers have a passive chassis, either NASA/JPL's rocker-bogie chassis or the 3-bogie chassis of the ExoMars. The goal of both suspension systems is to keep all six wheels on ground even for highly structured surfaces and distribute the gravitational forces as equally as possible. Some planetary rovers with an articulated, active suspension, similar to the MMX rover, were engineered and tested on earth, but have not been part of any mission. For example, JPL's Sample-Return Rover (SRR) is a four wheeled rover but with steerable wheels and only two shoulder motors that are each actuating the two legs on one side of the rover at the same time [5]. On this platform, a behavior-based control strategy for an enhanced stability on rough terrain was developed. Further locomotion studies were made by KANG et. al. on a six wheel robotic vehicle with an articulated suspension [6]. Its curves are driven via the skid steering method, since the wheels are not steerable themselves.

The ExoMars rover has a triple-bogie suspension with six wheels, capable of wheel-walking. In [7], the performance of wheel-walking was compared to normal rolling and enhanced locomotion capabilities were shown. Similar to the wheel walking is the inching locomotion, which was implemented and studied by MORELAND et al. [8] on Scarab, a four wheeled rover with an active suspension. They observed increased net traction resulting from a lower wheel motion resistance and a higher thrust of the stationary wheels. Inch-

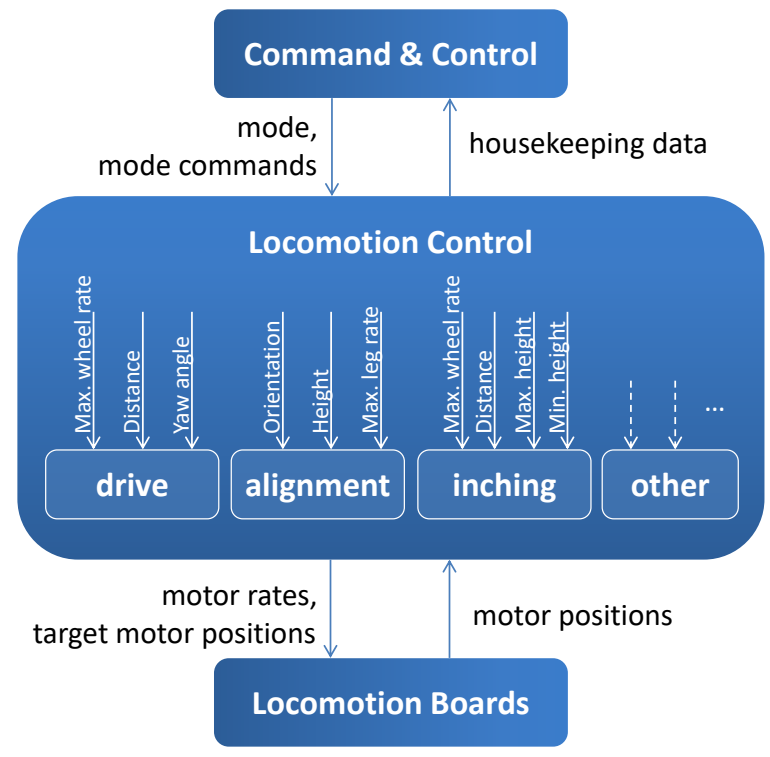

Figure 2: Control architecture: The main interfaces of the locomotion control software and the inputs for the individual modes.

ing locomotion, as well as a body alignment function, was also implemented on DFKI's wheel-legged rover Sherpa [9]. It has six degrees of freedom at each leg, allowing for a high flexibility in rover locomotion, alignment and ground adaption.

\section{Locomotion Control Algorithm}

The locomotion control algorithms are serving as control allocation between a high-level motion control and a lowlevel actuator control.

Within the overall rover software architecture, the locomotion control is embedded between the command control and the locomotion boards, see figure 2. The command control is the central software on the OBC managing and scheduling the rover operations. The locomotion electronic boards control the motors and convert analogue sensor signals. The demanded inputs for the motors are the desired maximum motor rate as well as the final motor position. Hence, a motor rate $\dot{\theta}$ and a motor position difference $\Delta \theta$ are computed for each motor in all of the control functions. The motor position difference will be added to the current motor position to obtain the motor target position. The calculated motor rate is a maximum motor rate to which the actual motor rate is ramped up within the motor controller. The motor control is implemented on a FPGA on the locomotion electronic boards and is not discussed further in here, see [2] for more details. The communication between locomotion control and the locomotion boards runs with a frequency of $10 \mathrm{~Hz}$. In addition to the motor commands, the locomotion control can send commands to enable or disable single motors and send motor soft reset signals.

The inputs for the locomotion control are the desired locomotion mode as well as its individual input parameters depending on that locomotion mode. These individual inputs are described in more detail in the specific subsections. 
The locomotion control algorithms for the MMX rover are developed under consideration of the requirements on the locomotion on the one hand and the restrictions by the rover design on the other hand. Motor hall sensors are integrated by the electronic boards such that the delta position from the startup of the boards is available. Moreover, each shoulder joint is equipped with two redundant potentiometers which provide the absolute leg positions relatively to the rover body. Other sensor values, e.g. the gravitation vector as well as the absolute rover position and its orientation are not available in sufficient extent for the locomotion control. The rover orientation is only estimated by the rover's shoulder joint positions. Thus, the control algorithms are taking the motor position feedback into account, but they are not controlling the setpoints on base of this feedback.

In the baseline version of the locomotion control algorithms, presented in this paper, the terrain is assumed to be locally flat. These assumptions very likely will not reflect the surface encountered on Phobos, nevertheless with available sensors, the rigid chassis and short development of the rover more complex algorithms using some kind of terrain adaption is not planned in this version and will be evaluated in the future.

In the following subsection, the wheel slip compensation that is relevant in some of the locomotion modes is represented. Afterwards, the individual modes are explained in detail.

\section{Wheel slip compensation}

The wheel slip compensation is not a locomotion mode itself, but a necessary computation for several locomotion modes, namely the uprighting mode, the alignment mode and the inching mode. Its purpose is that the wheels are not dragged over the ground while the shoulder joints are rotating the leg, but roll with zero slip. In the slip compensation algorithm, a wheel motor angular position difference $\Delta \tilde{\theta}$ and a wheel rate $\tilde{\dot{\theta}}$, that are added to the actual wheel velocity- and positioncommands, are computed. For both values, the plane in which the wheels are positioned at the beginning of the movement, in the following called wheel plane, is relevant. This is supposed to be the plane in which the movement is taking place and it is calculated via the measured leg angles and the rover kinematics.

For the motor angular position difference, the distance between the wheel positions at the current leg angle position and the target leg angle position is computed. It is projected on the wheel plane to get the distance $d_{W}$ the wheel actually has to cover, see figure 3 . The angle by which the wheel needs to rotate is then yielded by

$$
\Delta \tilde{\theta}=\frac{d_{W}}{r}
$$

where $r$ denotes the wheel radius.

To obtain the wheel rate, the wheel velocity relative to the rover body is calculated via the shoulder motor rate. For the wheel slip compensation, only the component in the plane in that the leg is turning, is relevant. This one is projected onto the wheel plane, see figure 3 , to receive the velocity of the wheel $\dot{d}_{W}$. The final value is then obtained by

$$
\tilde{\dot{\theta}}=\frac{\dot{d}_{W}}{r}
$$

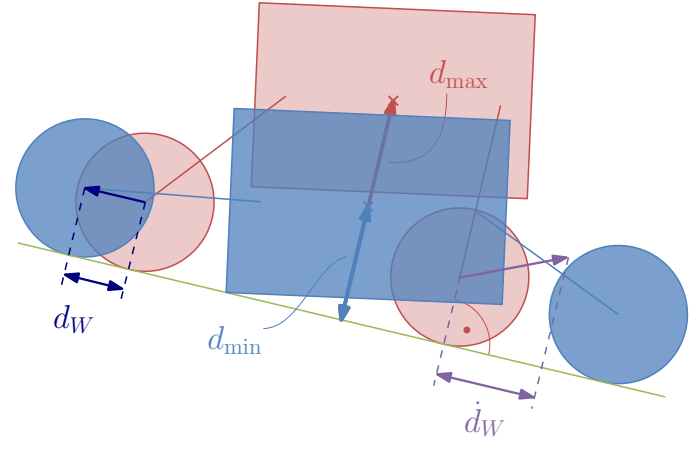

Figure 3: Wheel distance and velocity for the slip compensation and minimum and maximum feasible distances for a certain orientation.

\section{Driving}

On system level, the rover is required to drive forward, in curves and accomplish point turns. These movements are realized in the driving mode through skid steering, since the wheels are not steerable. Thereby, the wheels must turn with coordinated speeds. To prevent undesired rotations of the legs, in particular in the case of strong forces acting on them, the leg motors are kept active even though no action is required.

A drive command consists of three inputs, see figure 4:

- the position difference from the current rover position to its target position along a circular track, expressed by $\Delta x_{\text {des }}$,

- a heading angle difference between the current rover yaw orientation and the target yaw orientation, expressed by $\varphi_{\text {des }}$, and

- a maximum wheel rate $\dot{\theta}$ to determine the speed of the operation as explained further below.

The angular position difference of the wheels is computed by

$$
\Delta \theta=\frac{\Delta x_{\mathrm{des}}-\frac{w}{2} \cdot \varphi_{\mathrm{des}}}{r}
$$

where $w$ denotes the rover width. The wheel rate input is used as the maximum wheel rate, because the wheel rates on the left and right side of the rover differ from each other in curves. The final motor rate command $\dot{\theta}$ for the individual motors is computed by the maximum duration that the wheels need to get with the demanded maximum rate to the desired position:

$$
\begin{aligned}
t_{\max } & =\max _{i=1, \ldots, 4}\left(\frac{\left|\Delta \theta_{i}\right|}{\dot{\theta}_{\mathrm{des}}}\right) \\
\dot{\theta}_{i} & =\frac{\Delta \theta_{i}}{t_{\max }}
\end{aligned}
$$

where $i$ indicates the wheel and $t_{\max }$ denotes the maximum duration of the commanded operation. Thus, all of the four wheels reach their target at the same time and a uniform movement over the entire action is ensured.

For straight driving, for a rover forward velocity of $v_{R \text {, des }}$ under zero slip assumption, the wheel rate that has to be commanded can be calculated by

$$
\dot{\theta}=\frac{v_{R, \text { des }}}{r},
$$




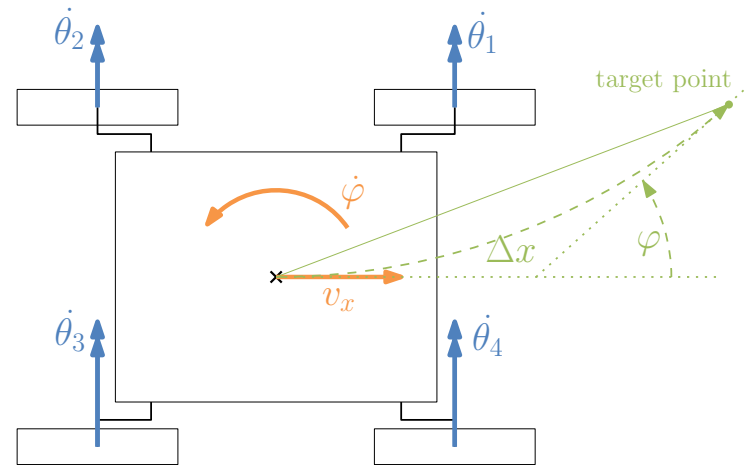

Figure 4: Drive mode: The maximum wheel rate and the target position are defined by the input, the wheel rates are computed by the locomotion control software.

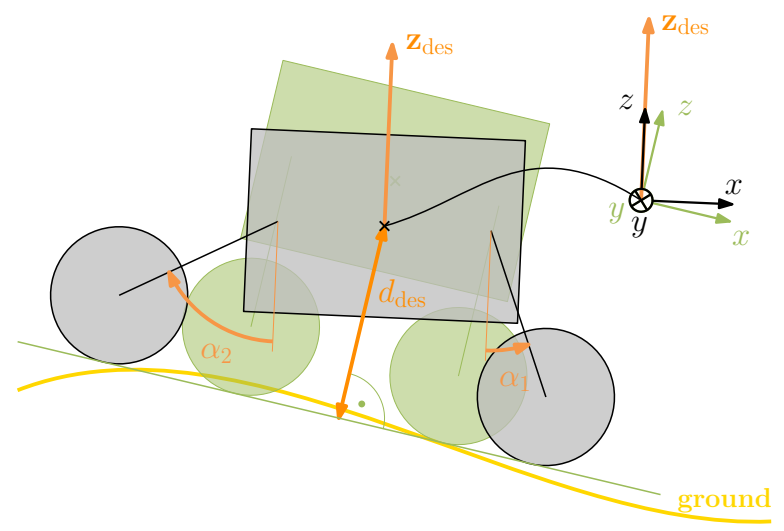

Figure 5: Definition of the variables for the alignment control: reference plane, orientation vector and vertical distance. Note, that the true ground geometry (yellow) is not known to the locomotion software.

where $v_{R \text {, des }}$ denotes the desired rover forward velocity and $r$ the wheel radius. Because of the low gravity on Phobos, the rover is supposed to drive with a default velocity of $1 \mathrm{~mm} / \mathrm{s}$ and a maximum velocity of $10 \mathrm{~mm} / \mathrm{s}$. For faster rover velocities, the vertical components of the ground contact forces would cause the rover to lift off.

\section{Alignment}

The aim of the alignment mode is to alter the orientation and the rover height primarily by adjusting the four leg angles. To enable stability, all four wheels are required to be on one plane. This leaves the distance of the rover to that plane, in the following called reference plane, as remaining degree of freedom. For feasible configurations, there are generally two possible leg angles, since the leg can either be rotated towards the other wheel of the respective side or away from it. Again, to enable or enhance stability, the outward solution is chosen. Taking this additional criterion into account, the problem is fully specified by commanding the following two quantities, see black rover in figure 5:

1. The desired rover $\mathbf{z}$-axis vector $\mathbf{z}_{\mathrm{des}}$ to define the desired rover orientation and

2. the desired distance $d_{\text {des }}$ between the rover origin and the reference plane.

At this point, it should be noted that the locomotion control has no knowledge of the actual ground and contact geometry.
For an unambiguous description of the above quantities, a reference coordinate system (RCS) is introduced (green in figure 5), whose origin is congruent with the origin of the rover body coordinate system (BCS, black in figure 5). The orientation of the RCS represents the upright oriented rover, which means that RCS and BCS are identical if all legs have the same angle, e.g. straight down as sketched in green in figure 5 .

For all following computations, the desired vertical axis of the rover, which is described in the RCS, is used to calculate the plane normal of the desired wheel plane, described in the BCS:

$$
\mathbf{n}_{\mathrm{des}}=\frac{1}{\left\|\mathbf{z}_{\mathrm{des}}\right\|}\left[\begin{array}{c}
-z_{\mathrm{des}, x} \\
-z_{\mathrm{des}, y} \\
z_{\mathrm{des}, z}
\end{array}\right] .
$$

Before the actual leg angles for the desired pose of the rover are computed, it must be ensured, that the combination of inputs $\mathbf{z}_{\mathrm{des}}, d_{\mathrm{des}}$ is feasible. Therefore, the desired orientation $\mathbf{z}_{\text {des }}$ is taken, and the minimum and maximum feasible distances $\left[d_{\min } ; d_{\max }\right]$ are searched, see figure 3 . The minimum allowed distance is defined as one of the corners of the rover body box touching the ground, see blue rover in figure 3, which can be calculated with a dot product as

$$
d_{\min }=\max _{i=1, \ldots, 4}\left(-\left\langle\mathbf{r}_{\mathrm{c}_{i}} ; \mathbf{n}_{\mathrm{des}}\right\rangle\right) .
$$

Therein, $\mathbf{r}_{\mathrm{c}_{i}}$ is the vector from the BCS origin to the $i$ th corner of the box ground plate, $\mathbf{n}_{\text {des }}$ is the desired plane normal and $\langle\cdot ; \cdot\rangle$ describes the dot product. The maximum allowed distance is reached, when at least one leg length projection onto the desired plane normal has reached its maximum, see red configuration in 3 . To calculate this projected length, it needs to be taken into account, that all legs can only move in a plane parallel to the $x$-z-plane of the BCS, see figure 6 . Therefore, the associated desired plane normal component in the leg plane is normalized as

$$
\mathbf{n}_{\mathrm{des}, \mathrm{L}}=\left\|\left[\begin{array}{c}
n_{\mathrm{des}, 1} \\
0 \\
n_{\mathrm{des}, 3}
\end{array}\right]\right\|^{-1}\left[\begin{array}{c}
n_{\mathrm{des}, 1} \\
0 \\
n_{\mathrm{des}, 3}
\end{array}\right] .
$$

The distance $\mathbf{r}_{\mathrm{s}_{i}}$ between BCS origin and each shoulder is projected onto the desired plane normal,

$$
b_{i}=\left\langle\mathbf{r}_{\mathrm{s}_{i}} ; \mathbf{n}_{\mathrm{des}}\right\rangle,
$$

and the maximum distance results in

$$
d_{\max }=\min _{i=1, \ldots, 4}\left(-\left\langle\mathbf{n}_{\mathrm{des}} b_{i}-l_{l} \mathbf{n}_{\mathrm{des}, \mathrm{L}}-r ; \mathbf{n}_{\mathrm{des}}\right\rangle\right)
$$

where $l_{l}$ denotes the leg length, and $r$ the wheel radius. Having the minimum and maximum distance available, it is checked if $d_{\mathrm{des}} \in\left[d_{\min } ; d_{\max }\right]$. If this interval is empty, the orientation is infeasible for any distance.

For feasible inputs $\mathbf{z}_{\mathrm{des}}, d_{\mathrm{des}}$, the absolute leg angles to achieve this desired pose can now be computed with geometric relations, see figure 6 . To make the calculations easier, the wheel is assumed to have contact with the ground in one point straight under the wheel hub as shown in the sketches. First, the distance from the shoulder joint to the desired plane through the wheel hubs is calculated as

$$
h_{i}=-\left(b_{i}+d_{\mathrm{des}}-r\right) .
$$

This distance now needs to be projected onto the desired normal component in the leg plane $\mathbf{n}_{\mathrm{des}, \mathrm{L}}$, which yields $\bar{a}_{i}$. 


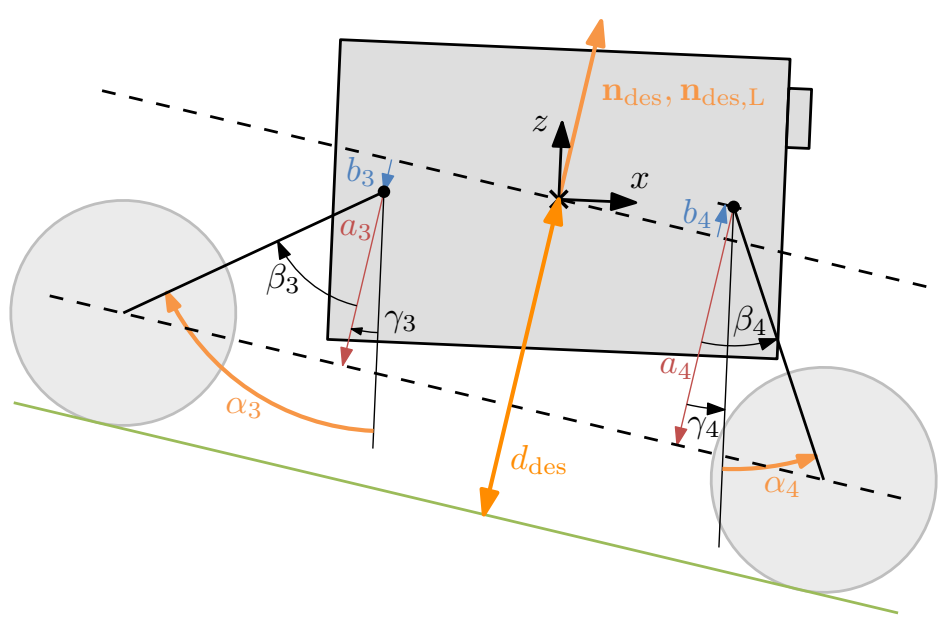

(a) side view

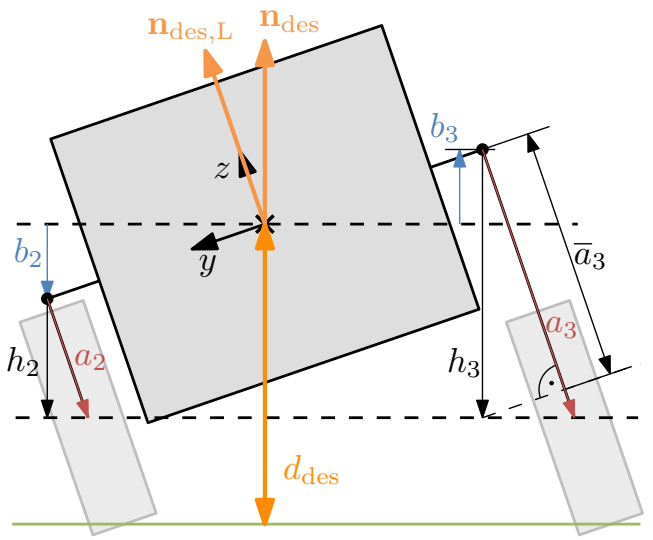

(b) rear view

Figure 6: Geometric relations for the leg angle computation.

The dimension of interest is the required distance between shoulder joint and wheel hub in the direction of $\mathbf{n}_{\text {des,L }}$, which results from $\bar{a}_{i}$ through geometric similarity as

$$
\frac{a_{i}}{h_{i}}=\frac{h_{i}}{\bar{a}_{i}} \Leftrightarrow a_{i}=\frac{h_{i}^{2}}{\left\langle h_{i} ; \mathbf{n}_{\mathrm{des}, \mathrm{L}}\right\rangle} .
$$

From here, the absolute leg angles $\alpha_{i}$ can be composed as

$$
\begin{aligned}
\alpha_{i} & =\beta_{i}+\operatorname{sign}\left(-n_{\operatorname{des}, \mathrm{L}, x}\right) \gamma_{i} \\
& =\operatorname{acos}\left(a / l_{l}\right)+\operatorname{sign}\left(-n_{\operatorname{des}, \mathrm{L}, x}\right) \operatorname{acos}\left(\left\langle\mathbf{n}_{\operatorname{des}, \mathrm{L}} ; \mathbf{e}_{z}\right\rangle\right) .
\end{aligned}
$$

Therein, the angles $\alpha_{i}, \beta_{i}, \gamma_{i}$ are defined as shown in figure 6 and $\mathbf{e}_{z}$ is the unit vector of the BCS's $z$-axis. It can be seen in figure 6a, that each of the angles $\beta_{i}$ could either be positive or negative if the only requirement is that all wheels should be on one plane. However, as stated in the beginning of this section, only the configuration that shows away from the other wheel is of interest, i.e. only the positive solutions for the angles $\beta_{i}$ are taken into account. The angle $\gamma_{i}$ between the desired plane normal and the rover's $z$-axis needs to be added or substracted, depending on the configuration, which is achieved with the signum function in (14).

The leg rates are calculated according to their total angle to rotate and a maximum leg rate analogously to the wheel rates in (4) and (5). This leads to a coordinated adjustment of the rover body orientation with all leg movements being finished at the same time. The wheels need to be turned as well for zero slip rolling as it was described in the section Wheel slip compensation.

\section{Inching locomotion}

The intent of the inching locomotion is a maximization of the traction and a minimization of slippage and leads therefore to a lower risk of wheel sinkage. It is indended to be used on hazard terrain and the rover is supposed to move only straight forward. Thereby, the front and rear wheel pair move alternately and at the same time the rover body moves up and down by turns, which looks like a moving inchworm. During this process, the rover body is determined to be parallel to the reference plane which was defined in the previous subsection. Hence, the inching locomotion mode requires as input parameters:

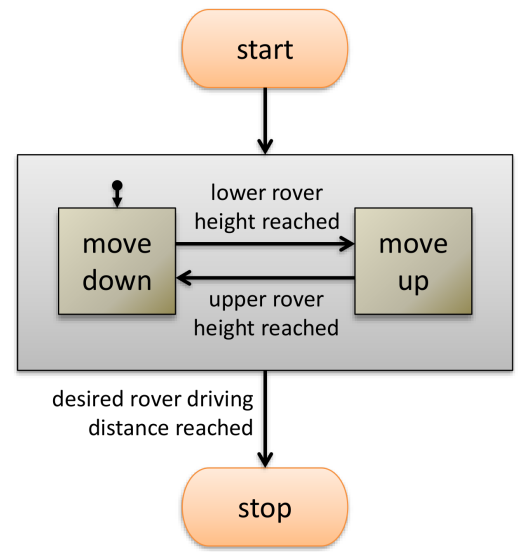

Figure 7: The process of the inching locomotion.

- a lower and upper rover height that the rover shall be positioned in during the operation of the inching locomotion - the total distance that shall be driven in the execution of the inching mode and

- the maximum wheel velocity during this time.

The alternating movement is realized by a state machine, see figure 7. The start procedure contains initial calculations and the initialization of the rover in its upper position. From there on, the front and rear drive trains are moving alternately until the target position is reached. As initial calculations, the target leg angles in the requested minimum and maximum body height are calculated by

$$
\alpha=\arccos \left(\frac{d_{S}-r}{l_{l}}\right),
$$

where $d_{S}$ denotes the distance between the shoulder joint and the reference plane, $r$ the wheel radius and $l_{l}$ the length of a leg. Since the rotational directions of the wheels are defined as in figure $4, \alpha$ is multiplied by -1 for the front wheels, so that the legs stir out from the rover body. The target wheel angles are calculated under the assumption of zero slip, similar to equation 3 , via

$$
\Delta \theta=\frac{\Delta x_{\mathrm{des}}}{r} .
$$




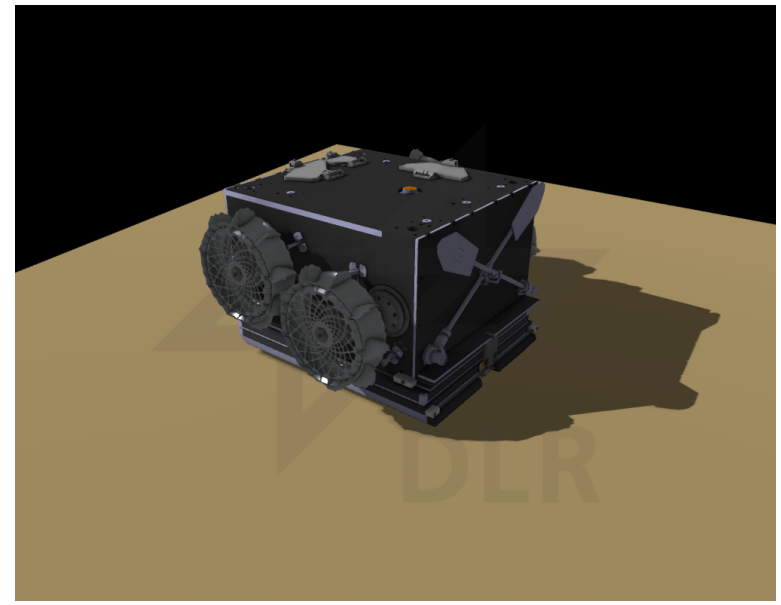

Figure 8: The cruise positioning of the legs and flaps. In this situation, the rover was landed on its upper side, i.e. on the folded solar panels. Hence, in this figure, one can see the right side, the bottom side and the back of the rover.

During the states "moving up" and "moving down", the respective wheel and leg rates are calculated. Thereby, the leg rates are always the commanded maximum leg rates, but with opposed signs depending on the moving direction. The wheel rates are calculated so that the not moving wheels stay at their position with no rotation relative to the world and the moving wheel pair rolls with zero slip. The state machine stops when the target position is reached, i.e., when the wheels have reached their initially calculated target angle.

\section{Uprighting and pass-through mode}

The uprighting procedure is the first task that the locomotion system of the MMX rover will fulfill on Phobos. During cruise, the legs are in a position so that the rover has its minimal possible volume expansion, see figure 8 . The process to get from this cruise position into an inertial position from which the solar panels can be unfolded will be called uprighting. The uprighting process is supported by two movable flaps on the rear side of the rover body, controlled by the locomotion control. Depending on the side the rover lands on, a particular sequence, in which order the respective leg and flap motors are actuated, will be commanded to the locomotion control system. It is provided by CNES and not elaborated in this paper. Nevertheless, this operation is different from the others in certain aspects. The uprighting mode will be the only mode where the flap motors are activated, excepting the special pass through mode that will be described further below. Moreover, the leg position is not restricted since the ability to actively perform a full turn of the legs is essential for this task. Since only flap and leg velocities and positions are commanded, respectively, the wheel positions and commands will be calculated by the locomotion control software such that the wheels roll with no slip as it was described in the section Wheel slip compensation. Since the testing platform introduced in section 4 is not made for uprighting tests, those were only running in simulation but are not represented in here.

The pass through mode is for testing before launch and during mission for critical situations in which single motor commands are given from teleoperation of the rover. Motor commands are directly given in absolute positions, be checked for validity and passed through to the motors.

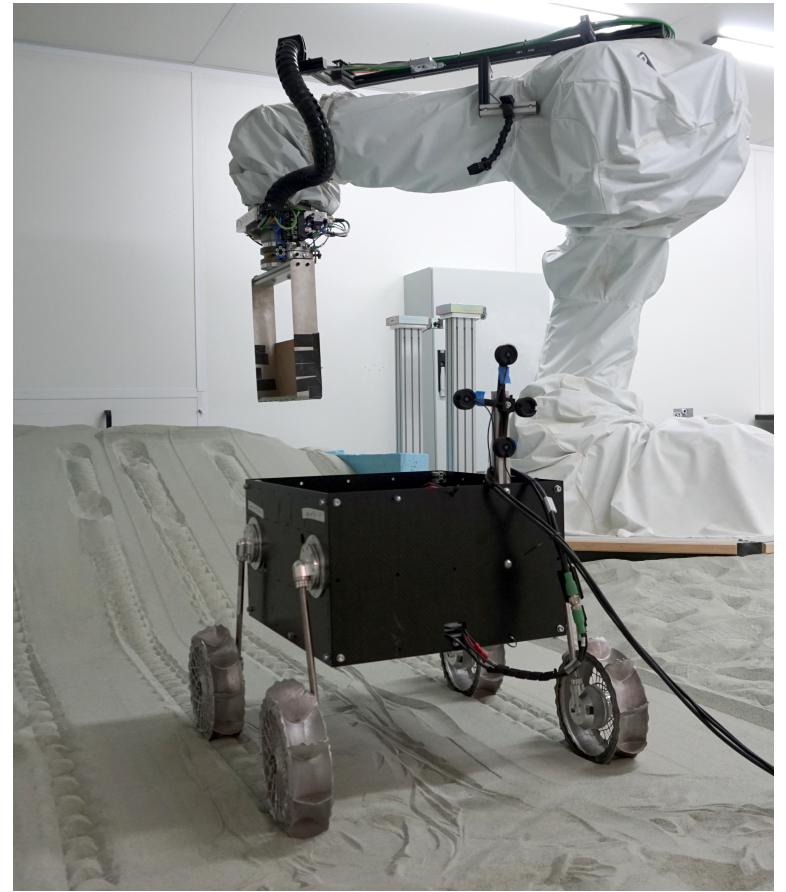

Figure 9: The MMX prototype in the olivine sand in the front and the TROLL robot arm in the back.

\section{Testing, Results And Discussion}

To verify and evaluate the implemented algorithms, a locomotion test campaign was performed, for which DLRRMC (Robotics and Mechatronics Center) assembled a rover prototype, see figure 9 . This prototype includes solely the locomotion subsystem according to the current design and necessary subsystems that were replaced by a simpler version that suits the locomotion subsystem's needs, such as the box that represents the rover chassis. Following that approach, the prototype consists of:

- a CFK chassis whose dimensions are similar to those of the final rover

- legs and wheels with their drive trains in the current stage of development,

- the locomotion electronic boards that combine motor controller, communication interface and ADC converter for the analogue sensor signals,

- a power inverter board that provides the voltage levels that will later in the mission be provided by the Power Distribution Control Unit (PDCU) and

- an interface board, that allows direct access to the SpaceWire link of the locomotion electronics from a Linux computer via USB.

The first design of the locomotion control software, that is used within this test campaign, was implemented and validated in MATLAB/Simulink [10]. The communication from the controller in MATLAB/Simulink to the locomotion boards was realized via a serial communication. With this setup, it was possible to test the rover's locomotion performance with limited availability of the remaining components.

The tests were performed in the Terramechanics Robotics Locomotion Lab (TROLL) [11] on three different terrains. As soil simulants, two variants were chosen: a rough lava sand (called RMCS12 in [11]) and a fine olivine sand (called 
RMCS14 in [11]). The rough lava sand is a large-grained cohesion-less sand. Due to its high traction capabilities, it is particularly interesting for test cases where high structural loads could be an issue. The olivine (RMCS14) was chosen as a baseline soil simulant, since its small grain size and cohesion-less properties provide moderate traction. For the analysis of the system performance on hard ground, a hard wooden board was used. These testing conditions were chosen to cover a wide range of possible Phobos surfaces. A more specific choice of simulants is not beneficial due to lacking knowledge about the surface conditions of Phobos and the considerable influence of the low gravity on the soil behavior. Due to the latter, the performed test can only be treated as a functional and qualitative testing, not being representative of the quantitative performance on Phobos.

For this test campaign, the key feature of the TROLL laboratory is the robot arm which is capable of automatically and repeatably prepare the soil in the test area, see figure 9. Therewith, the sand was loosened and leveled before each test run. As specified in the mission requirements, slopes of up to $10^{\circ}$ are to be expected in the landing area. To test the systems capabilities further, slopes of up to $20^{\circ}$ were tested.

To capture the rotation angle of wheels and legs, the integrated hall encoders values were logged from the locomotion boards. Additionally, a k-series optical camera [12] allowed position and orientation tracking of the rover for a performance analysis and conventional cameras were used for analysis of the qualitative behavior. A logging rate of 6 $\mathrm{Hz}$ was deemed appropriate due to the slow movement of the rover.

\section{Driving forward, backward and inching}

Driving forward and backward are two essential functionalities of locomotion. While the rover chassis itself is symmetrical, the wheels are not, see figure 9 or [2]. Their grousers are formed convex which leads to the assumption that forward and backward driving perform differently.

Setting: For driving forward and backward, the drive mode was used. To compare the drive performance on the different terrains and slopes, the rover was commanded to drive straight forward and backward for a length of $0.4 \mathrm{~m}$ (about one rover length) with a rover velocity of $0.1 \mathrm{~m} / \mathrm{s}$. The total driven distance was measured and used to calculate the slip as

$$
\text { slip }=1-\frac{\text { driven distance }}{\text { commanded distance }} .
$$

Note that the actual command values are the delta wheel angles, which are calculated from the desired distance with an assumed radius since the effective radius is not known, see equation 1 . The measured slip values in percent are summarized in the first two columns of table 1.

Straight driving is compared to the inching locomotion in the olivine sand and on slopes of $0^{\circ}, 10^{\circ}$ and $20^{\circ}$. The inching locomotion was performed with these input values:

- maximal wheel rate $=0.1 \mathrm{rad} / \mathrm{s}$

- distance $=1 \mathrm{~m}$

- minimal height $=0.24 \mathrm{~m}$

- maximal height $=0.29 \mathrm{~m}$.

The slip values are shown in the last column of table 1 .

Three repetitions were done for each test to ensure a repro- ducibility. The average slip value of the three tests in percent is depicted in table 1 and the standard deviation is added in parenthesis.

Table 1: Slip for straight driving forward, driving backward and inching in percent. Given are the mean values of three repetitions and the standard deviation in parenthesis.

\begin{tabular}{|l|r|r|r|}
\hline terrain & \multicolumn{3}{|c|}{ slip [\%] } \\
\& slope & forward & backward & inching \\
\hline \hline olivine $0^{\circ}$ & $5.4(0.4)$ & $-9.7(0.1)$ & $-6.5(2.6)$ \\
olivine $10^{\circ}$ & $16.4(2.6)$ & - & $16.0(1.6)$ \\
olivine $20^{\circ}$ & $36.5(1.0)$ & - & $32.5(0.7)$ \\
lava sand $0^{\circ}$ & $1.5(1.3)$ & $-8.3(2.1)$ & - \\
plate $0^{\circ}$ & $-18.2(0.9)$ & $-17.4(0.3)$ & - \\
\hline
\end{tabular}

Results: For straight forward driving with no slope, the slip value is below $10 \%$ on both of the sand simulants, which is relatively low for a rover on sandy terrain thanks to the chosen wheel design. On the hard ground, the slip values are even negative, which means that the rover traveled further than expected. This can be explained by the difference between the wheel rim radius, which is used in the controller, and the varying effective radius. This effective radius gets significantly larger on hard ground, where the rover drives on its grouser tips.

For backward driving, the slip becomes negative even on sandy terrain. This stems from the convex grouser shape, see figure 9, which shovels into the sand when driving forward, but lifts the wheel - provided the soil offers enough support - backward. However, this effect is expected only for low sinkage, since the soil in that case provides enough support to drive mainly on the convex grouser faces during backwards driving. In softer sands with more sinkage, the shoveling effect for forward driving is desired and provides better traction according to simulation. The result for the tested sand with low wheel sinkage is a larger effective radius when driving backward, yielding a lower, or even negative, slip. Additionally, all backwards drives were performed in the ruts of the respective forward drive. In earlier experiments it was found that, for low slip values, this multi-pass results in a more compressed soil, leading to less sinkage as well. On hard ground it can be seen that forward and backward slip values are quasi identical, which is sensible since the rover drives on the grouser tips with equal effective radius in either direction.

Compared to the rover slip during common straight forward driving, the inching locomotion mode has a similar performance when the rover drives on a flat ground or moderate slope. For the $20^{\circ}$ slope angle, the inching locomotion yields lower slip values than the normal driving mode.

\section{Driving curves and point turn}

Next to forward and backward driving, the driving mode enables curve drives and point turns. A set of experiments was carried out to compare the achieved yaw angle with respect to the commanded ones for different curve radii.

Setting: To achieve curves of different radii, a constant yaw angle command of $90^{\circ}$ is combined with different distances. Distances of $1.2 \mathrm{~m}$ and $0.6 \mathrm{~m}$ result in a wider and a narrower curve, respectively, while a point turn is performed with a commanded distance of $0 \mathrm{~m}$. The maximum wheel velocity 
for all experiments was set to $0.1 \mathrm{rad} / \mathrm{s}$. Note that the largest distance could only be driven in the olivine sand, since the wooden plate and the lava sand bin were both shorter. Additionally, the turning in lava sand has shown excessive forces and torques on the mechanics of the locomotion subsystem. Therefore, only one run was performed per setting in the lava sand.

The total achieved yaw angles were measured with the tracking system and the relative errors are calculated by:

$$
\text { relative error }=\frac{\text { commanded value }- \text { measured value }}{\text { commanded value }} \text {. }
$$

The results are summarized in table 2 .

Table 2: Relative yaw angle errors for point turn and different driving distances in percent. Given are the mean values of three repetitions and the standard deviation in parenthesis.

\begin{tabular}{l|r|r|r|}
\hline & \multicolumn{3}{|c|}{ relative yaw error [\%] } \\
terrain & 0m (point turn) & $0.6 \mathrm{~m}$ & $1.2 \mathrm{~m}$ \\
\hline \hline olivine & $48.0(2.3)$ & $54.5(0.6)$ & $45.2(0.5)$ \\
lava sand & $55.2^{*}$ & $57.8^{*}$ & - \\
plate & $34.1(0.4)$ & $32.9(2.8)$ & - \\
*: only performed once due to high mechanical stress on the \\
chassis
\end{tabular}

Results: The yaw angle error is very high and ranges between $30 \%$ and $50 \%$ on the different surfaces. This was expected due to the skid steering approach, which ignores side forces on the wheels and simply calculates the wheel rates with a longitudinal no-slip assumption. It was assumed that decreasing the side slip by increasing the curve radius would decrease the resulting side slip and thereby side forces at the wheels and thereby the yaw angle error. While a clear trend from the point turn to the $0.6 \mathrm{~m}$ distance (first two columns of 2 , respectively) cannot be seen, the $1.2 \mathrm{~m}$ distance yields lower error. However, a distinct relation cannot be deduced, therefore, further analysis will be performed to address this question.

\section{Alignment}

To test the alignment mode, two different scenarios were tested. Firstly, the rover body was lowered and lifted by $10 \mathrm{~cm}$, while it was kept parallel to the ground. Secondly, the rover was commanded to perform a pitch movement which is reflected in an orientation vector of $[0.2,0,1]$ relative to the reference plane.

The actual movement was determined with the tracking system and compared to the respective target value. The relative error was calculated through (18) and the results are shown in the tables 3 and 4 .

Results: While the errors are considerably lower than for curve driving, they are still non-neglectable. All errors in the body lifting experiment are negative, which means that the desired delta body height is over-achieved. As for the driving experiments, this stems from the varying effective radii of the wheels. The variance within repetitions was a bit higher on hard ground since the grouser position at initial and final orientation plays a crucial role for the actual contact point of the respective wheel. The rather high error for the downwards movement in olivine should be investigated in future test campaigns, since this test was at the boarder of the tracking
Table 3: Relative error for body lifting in percent with negative values indicating over-achievement. Given are the mean values of three repetitions and the standard deviation in parenthesis.

\begin{tabular}{|l|r|r|}
\hline & \multicolumn{2}{|c|}{ relative height error [\%] } \\
terrain & down & up \\
\hline \hline olivine & $-11.0(2.3) * *$ & $-4.0(0.5)$ \\
lava sand & $-6.5(1.1)$ & $-1.5(0.4)$ \\
plate & $-6.5(2.4)$ & $-7.5(2.6)$ \\
\hline \multicolumn{2}{|c|}{$* *:$ only two valid experiments }
\end{tabular}

Table 4: Relative error for a pitch movement in percent. Given are the mean values of three repetitions and the standard deviation in parenthesis.

\begin{tabular}{|l|r|r|}
\hline \multirow{2}{*}{ terrain } & \multicolumn{2}{|c|}{ relative pitch error [\%] } \\
pitch forward & back to upright \\
\hline \hline olivine & $-8.6(4.2)$ & $-9.1(2.1)$ \\
lava sand & $0.1(2.2)$ & $-4.3(0.9)$ \\
plate & $-3.3(1.3) * * *$ & $1.4(0.2) * * *$ \\
\hline
\end{tabular}

range. Therefore, larger tracking errors might occur and only two of the three repetitions gave valid measurements.

\section{Discussion}

A specific interpretation of the results was made for each set of experiments and explanations were given. Consolidated, it can be seen that the deviations from commanded values, i.e. the slip, the yaw angle error and the pitch and height error, vary considerably. These variations stem from the wheel ground contact situation, i.e. the irregular shape of the wheel with its large grousers, see figure 9, and varying ground conditions. To be able to reduce these errors, information about the ground and the contact situation would need to be known or estimated on-board the rover. Since this is not feasible with the available sensor suite and no reliable framework exists for that, the errors must be accepted. In fact, the first drives on Phobos will be used to tune a full rover simulation on ground, which in turn will then predict the behavior of the MMX rover. With the help of this operations simulator, the engineers and operators will then choose the motion commands according to the expected error level.

\section{Conclusion}

Based on the requirements on the locomotion subsystem and considering the design of the rover chassis, locomotion control allocation algorithms for the MMX rover were developed. The details of the different locomotion modes were explained and presented by means of the kinematic equations.

For a qualitative analysis, tests were performed with a prototype on different grounds. It was shown that the rover slip is below $10 \%$ on the tested terrains due to the optimization of the wheel for high traction. Differences between forward/backward driving, different grounds and slopes were shown. Additionally, a mode for soft sanded slopes, the inching locomotion, has shown to decrease slip for a $20^{\circ}$ slope by about $10 \%$. Body lifting and orientation modes perform well on flat ground. However, the individual ori- 
entation of grousers and the resulting contact situation leads to errors of up to $10 \%$ for these modes as well. The largest errors of $30-50 \%$ were seen for commanded yaw angles. This was expected, since the purely kinematic skid steering approach neglects side forces at the wheels, while assuming zero longitudinal slip.

Further tests, especially of the curve driving and inching locomotion, will be performed on different terrains to improve the chassis control software. The inching locomotion mode will be further refined, e.g. similar to the extensive ExoMars rover wheel-walking study of WIESE [13]. For enhanced curve driving, an adjustment of single wheel speeds is considered. However, it must be pointed out again, that all tests that are performed under earth gravity can only be used for comparative and qualitative analysis, since the effects of Phobos' milli-gravity cannot be achieved on earth. Therefore, extensive simulation models were developed, which are and will further be used throughout the mission.

Next steps within the MMX mission is the implementation and testing in $\mathrm{C}$-Code and on a representative $\mathrm{OBC}$ architecture.

\section{REFERENCES}

[1] S. Ulamec, P. Michel, M. Grott et al., "A rover for the JAXA MMX Mission to Phobos,” 2019.

[2] H.-J. Sedlmayr, S. Barthelmes, R. Bayer et al., "MMX - Development of a Rover Locomotion System for Phobos," in 2020 IEEE Aerospace Conference, 2020.

[3] A. Seeni, B. Schäfer, and G. Hirzinger, "Robot Mobility Systems for Planetary Surface Exploration - State-ofthe-Art and Future Outlook: A Literature Survey," pp. 189-208, Jan. 2010.

[4] A. Ellery, Planetary Rovers: Robotic Exploration of the Solar System. Berlin, Heidelberg: Springer Berlin Heidelberg, 2016, ch. Survey of past rover missions, pp. 59-69.

[5] P. S. Schenker, P. Pirjanian, J. Balaram et al., "Reconfigurable robots for all-terrain exploration," in Sensor Fusion and Decentralized Control in Robotic Systems III, G. T. McKee and P. S. Schenker, Eds., vol. 4196, International Society for Optics and Photonics. SPIE, 2000, pp. $454-468$.

[6] J. Kang, W. Kim, J. Lee, and K. Yi, "Design, implementation, and test of skid steering-based autonomous driving controller for a robotic vehicle with articulated suspension," Journal of Mechanical Science and Technology, vol. 24, no. 3, pp. 793-800, 2010.

[7] M. Azkarate, M. Zwick, J. Hidalgo Carrió et al., "First Experimental Investigations on Wheel-Walking for Improving Triple-Bogie Rover Locomotion Performances," in Advanced Space Technologies for Robotics and Automation. 13th ESA/Estec Symposium on Advanced Space Technologies in Robotics and Automation (ASTRA), May 11-13, Noordwijk, Netherlands. inproceedings ASTRA, 2015.

[8] S. Moreland, K. Skonieczny, D. Wettergreen et al., "Inching locomotion for planetary rover mobility," in 2011 Aerospace Conference, 2011.

[9] F. Cordes, A. Dettmann, and F. Kirchner, "Locomotion modes for a hybrid wheeled-leg planetary rover," in 2011 IEEE International Conference on Robotics and
Biomimetics, 2011, pp. 2586-2592.

[10] MATLAB version 9.5.0.944444 (R2018b), The Mathworks, Inc., Natick, Massachusetts, 2018.

[11] F. Buse, T. Bellmann, R. Lichtenheldt, and R. Krenn, "The DLR Terramechanics Robotics Locomotion Lab," in International Symposium on Artificial Intelligence, Robotics and Automation in Space, 2018.

[12] Lastchecked: 2020-09-12. [Online]. Available: https://www.metric3d.de/

[13] T. Wiese, "3D Kinematic Modeling and Evaluation of Rough-Terrain Locomotion Modes for an ExoMars-like Mobility Subsystem," Master's thesis, 2017.

\section{BIOGRAPHY}

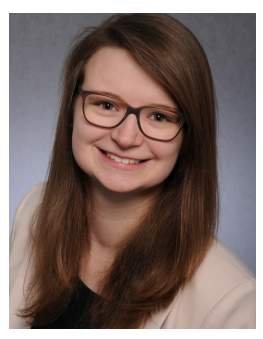

Juliane Skibbe studied Mathematics at the University of Würzburg and the Université d'Orléans. She wrote her Master's Thesis at the German Aerospace Center on the mathematical modelling of the compliant spokes for a rimless wheel rover. After finishing her Master's degree in 2018, she started working as a research associate at the Institute of System Dynamics and Control of the German Aerospace Center (DLR). Her main focus is on developing locomotion control algorithms for planetary exploration rover.

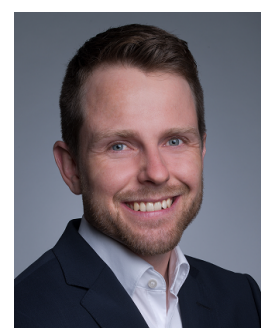

Stefan Barthelmes received his Dr.Ing. degree in Electrical Engineering at TU Darmstadt and his B.Sc. and M.Sc. degree in Mechanical Engineering from TU München. He is currently working as a research associate at the Institute of System Dynamics and Control of the German Aerospace Center (DLR). His main research focus is model-based chassis control and simulation model development of planetary exploration rovers. Within the MMX mission, he is responsible for the control software of the locomotion subsystem.

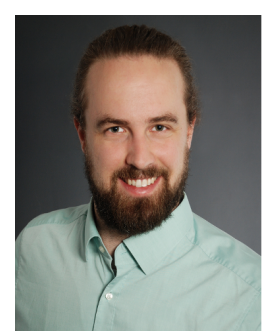

Fabian Buse received the degrees of B.Sc. and M.Sc. from RWTH Aachen University. Since 2015, he is a research associate at the Institute of System Dynamics and Control. His research interests are in terramechanics for planetary rovers. He is the lead engineer of the DLR Terramechanics Robotic Locomotion Lab (TROLL) and is leading the rover simulation for the MMX rover

project. 